\title{
The usefulness of fecal calprotectin in assessing inflammatory bowel disease activity
}

\author{
Yang Woon Lee, Kang-Moon Lee, Ji Min Lee, Yoon Yung Chung, Dae Bum Kim, Yeon Ji Kim, \\ Woo Chul Chung, and Chang-Nyol Paik
}

Department of Internal Medicine, College of Medicine, St. Vincent's Hospital, The Catholic University of Korea, Suwon, Korea

Received: October 5, 2016

Revised : February 24, 2017

Accepted: June 7, 2017

\section{Correspondence to}

\section{Kang-Moon Lee, M.D.}

Department of Internal Medicine, St. Vincent's Hospital, College of Medicine, The Catholic University of Korea, 93 Jungbu-daero,

Paldal-gu, Suwon 16247, Korea

Tel: +82-31-249-7138

Fax: +82-31-253-8898

E-mail:drmaloman@catholic.ac.kr
Background/Aims: Fecal calprotectin (FC) is known to correlate with disease activity and can be used as a predictor for relapse or treatment response in inflammatory bowel disease (IBD). We evaluated the usefulness of FC as a biomarker for disease activity in patients with IBD using both enzyme-linked immunosorbent assay (ELISA) and a quantitative point-of-care test (QPOCT).

Methods: Fecal samples and medical records were collected from consecutive patients with IBD. FC levels were measured by both ELISA and QPOCT and patient medical records were reviewed for clinical, laboratory, and endoscopic data.

Results: Ninety-three patients with IBD were enrolled, 55 with ulcerative colitis (UC) and 38 with Crohn's disease (CD). The mean FC-ELISA levels were 906.3 \pm $1,484.9 \mu \mathrm{g} / \mathrm{g}$ in UC and 1,054.1 $\pm 1,252.5 \mu \mathrm{g} / \mathrm{g}$ in CD. There was a strong correlation between FC-ELISA level and clinical activity indices $(p<0.05)$. FC-ELISA level was significantly lower in patients with mucosal healing $(\mathrm{MH})$ compared to those without MH in UC $(85.5 \pm 55.6 \mu \mathrm{g} / \mathrm{g}$ vs. $1,503.7 \pm 2,129.9 \mu \mathrm{g} / \mathrm{g}, \mathrm{p}=0.005)$. The results from the QPOCT corresponded well to those from ELISA. A cutoff value of 201.3 $\mu \mathrm{g} / \mathrm{g}$ for FC-ELISA and $150.5 \mu \mathrm{g} / \mathrm{g}$ for FC-QPOCT predicted endoscopic inflammation (Mayo endoscopic subscore $\geq 1$ ) in UC with a sensitivity of $81.8 \%$ and $85.8 \%$, respectively, and a specificity of $100 \%$ for both.

Conclusions: FC was strongly associated with disease activity indices, serologic markers, and endoscopic activity in patients with IBD. QPOCT can be used more conveniently than ELISA to assess FC in clinical practice.

Keywords: Calprotectin; Enzyme-linked immunosorbent assay; Quantitative point-of-care test; Inflammatory bowel diseases; Disease activity

\section{INTRODUCTION}

Mucosal healing $(\mathrm{MH})$ is reported to be associated with sustained remission and a reduced risk of surgery in inflammatory bowel disease (IBD) [1]. Colonoscopy is essential for the assessment of intestinal inflammation. However, the standard criteria for the evaluation of disease severity as well as the definitions of $\mathrm{MH}$ on colonoscopy are still unclear. Moreover, long-term studies examining whether $\mathrm{MH}$ actually contributes to remission maintenance in IBD are few [2-4].

Fecal biomarkers, such as calprotectin, are non-invasive and have been evaluated for the ability to assess disease activity in patients with IBD. Calprotectin is a calcium and zinc binding protein of the $\mathrm{S}-100$ protein family and is found in the cytosol of human neutrophils and macrophages. Calprotectin reflects the migration of neutrophils into the gut lumen and thus can be used as 
a sensitive marker of intestinal inflammation. Recently, several studies have demonstrated that fecal calprotectin (FC) may be the best available surrogate marker for the presence of mucosal inflammation in IBD [5-11].

FC is also known to have a good correlation with disease activity and may be a useful predictor for relapse or response to therapy in IBD. FC has been used as a follow-up marker in patients diagnosed with IBD. According to recent trials, the sensitivity of FC to detect any active mucosal disease ranges from $70 \%$ to $100 \%$ and the specificity ranges from $44 \%$ to $100 \%$, depending on the cut-off value used [12-14]. However, FC is not currently widely used in clinical practice in Korea.

The current method to measure FC is based on the enzyme-linked immunosorbent assay (ELISA). The ELISA has disadvantages such as being a time-consuming process, requiring expertise, and needing multiple samples at the same time in order to run the assay. However, the recently developed, quantitative point-of-care test (QPOCT) is simple, easy to use, and able to produce more rapid results than the ELISA. Unfortunately, there has been a lack of data comparing the accuracy and availability of the two methods [15,16].

In the current study, we aimed to assess the usefulness of FC as a marker of disease activity and examine the correlation between FC and laboratory and endoscopic parameters, including $\mathrm{MH}$, in patients with IBD. In addition, we compared the QPOCT with the established ELISA method.

\section{METHODS}

\section{Patients}

The prospective study was conducted at St. Vincent's Hospital of the Catholic University of Korea, between May 2012 and December 2014. Consecutive patients with a diagnosis of IBD of at least 6 months in duration were enrolled. The diagnosis of IBD was made on the basis of standard clinical, endoscopic, and histological criteria. Exclusion criteria were as follows: concomitant gastrointestinal infection within 4 weeks prior to enrollment, peptic ulcer disease within 2 months prior to enrollment, regular use of non-steroidal anti-inflammatory agents ( $\geq 2$ tablets/week), colorectal cancer, pregnancy, and an inability to collect fecal samples. The clinical phenotype was assessed according to the World Gastroenterology Organization's (WGO) Montreal Classification [17]. In ulcerative colitis (UC), the extent of the disease was classified as proctitis, left-sided colitis, or extensive colitis. In Crohn's disease (CD), the location of the disease was classified as ileal, colonic, or ileocolonic.

\section{Serological markers}

Serologic markers of inflammation including the white blood cell (WBC) count, C-reactive protein (CRP), and hematocrit were measured within 1 day before the collection of the fecal samples.

\section{Clinical activity}

In UC, clinical activity was assessed according to the partial Mayo score [18]. The partial Mayo score consists of three items: stool frequency, rectal bleeding, and the physician's global assessment. Clinical remission was defined as a partial Mayo score $\leq 2$ with a stool frequency subscore of o or 1 and a rectal bleeding subscore of $\mathrm{o}$.

In $\mathrm{CD}$, clinical activity was assessed according to the Crohn's disease activity index (CDAI) [19]. Clinical remission was defined as a CDAI score $<150$.

\section{Endoscopic activity}

Endoscopic activity was assessed according to the Mayo endoscopic subscore (MES) in patients with UC [18]. The MES reflects the severity of intestinal inflammation on colonoscopy as follows: o point, normal or inactive disease; 1 point, mild disease (erythema, decreased vascular pattern, and mild friability); 2 points, moderate disease (marked erythema, absent vascular pattern, friability, and erosions); and 3 points, severe disease (spontaneous bleeding and ulcerations). Endoscopic remission (i.e., $\mathrm{MH}$ ) was defined as an MES of o.

In patients with $\mathrm{CD}$, endoscopic activity was assessed by the simple endoscopic index for Crohn's disease (SES-CD). The SES-CD consists of four components: the presence and size of ulcers (none, score o; diameter 0.1 to $0.5 \mathrm{~cm}$, score $1 ; 0.5$ to $2 \mathrm{~cm}$, score $2 ;>2 \mathrm{~cm}$, score 3 ); the extent of the ulcerated surface (none, $0 ;<10 \%, 1 ; 10 \%$ to $30 \%, 2 ;>30 \%, 3$ ); the extent of the affected surface (none, o; $<50 \%, 1 ; 50 \%$ to $75 \%, 2 ;>75 \%, 3)$; and the presence and type of narrowings (none, o; single, can be passed, 1; multiple, can be passed, 2; cannot be passed, 3). The definition of endoscopic remission using the SES-CD is 
still obscure. Thus, with reference to a previous study, we defined endoscopic remission as an SES-CD score < 4 points $[20,21]$.

\section{Fecal calprotection}

Stool sample collection was performed 1 to 3 days before the endoscopy or outpatients visits. Stool samples ( 1 to $2 \mathrm{~g}$ per person) were collected and stored at $-20^{\circ} \mathrm{C}$ until analysis. FC was determined using a commercially available ELISA kit (Bühlmann Laboratories AG, Schönenbuch, Switzerland) and QPOCT kit (Quantum Blue; Bühlmann). The laboratory personnel conducting the analysis was blinded to the clinical history and the endoscopic findings of the patients.

In the FC-ELISA method, aliquots of approximately $100 \mathrm{mg}$ of feces were homogenized in $5 \mathrm{~mL}$ of extraction buffer. A $2 \mathrm{~mL}$ of the homogenate was then centrifuged in a microcentrifuge for 5 minutes. Then $3,000 \mathrm{~g}$ and $100 \mu \mathrm{L}$ of the diluted supernatant (1:50 with incubation buffer) were incubated at room temperature and placed onto a microtiter plate coated with a monoclonal capture antibody highly specific to the calprotectin heterodimeric and polymeric complexes. After incubation, washing, a second incubation with a specific detection antibody, and a further washing step, tetramethylbenzidine followed by a stop solution were added. The measuring range of the FC-ELISA is 10 to $1,800 \mu \mathrm{g} / \mathrm{g}$. The calprotectin cut-off level representing a positive value was $\geq 50 \mu \mathrm{g} / \mathrm{g}$ as stated by the manufacturer.

The FC-QPOCT relies on lateral flow assay technology and includes an easy to use reader system with a quantitative read out. There are two kinds of FC-QPOCT: the lower range Quantum Blue (LF-CAL) and the higher range Quantum Blue (LF-CHR). Both provide quantitative results within minutes, ranging from 30 to 300 or 100 to $1,800 \mu \mathrm{g} / \mathrm{g}$, respectively. All samples scoring > 300 $\mu \mathrm{g} / \mathrm{g}$ with LF-CAL were tested again with the LF-CHR kit in order to obtain the exact FC value.

\section{Statistical analysis}

The statistical analysis was carried out using the IBM SPSS version 20.0 (IBM Corp., Armonk, NY, USA). Numerical results are given as the mean \pm standard deviation and range except when specified. Student $t$ test, Mann-Whitney $U$ test, and an analysis of variance were used to compare means. Qualitative results were based on the chi-square test. The correlation analyses between FC, clinical activity, endoscopic activity and other serologic markers was based on the Spearman rank correlation coefficient $(r)$ for non-parametric correlations. The multivariate analysis consisted of multiple logistic regressions. In the comparison among three independent groups, the Kruskal-Wallis test was used. Receiver operator characteristic curves were constructed to find the best cutoff value for FC using the area under the curve (AUC). The best cutoff value was used to determine sensitivity and specificity. Differences with a $p<0.05$ were considered statistically significant. The correlation between FC-ELISA and FC-QPOCT was analyzed by Spearman correlation coefficient rank and interclass correlation index.

\section{Ethics statement}

This study was approved by the Institutional Review Board of the Catholic University of Korea (IRB No. VC12RISIo015). Written informed consent was obtained from all subjects after a full explanation of the project.

\section{RESULTS}

Initially, 106 IBD patients, 64 with UC and 42 with CD, were considered for enrollment. Among them, thirteen patients were excluded; 12 for carrying inappropriate stool samples, and one for having a concomitant gastrointestinal infection. Thus, a total of 93 patients (55 UC and $38 \mathrm{CD}$ ) between 18 and 85 years of age were finally enrolled.

Using FC-ELISA, it took about 2 hours to measure FC in 88 samples at once. Using FC-QPOCT, it took 12-15 minutes for each sample depending on the kit type (12 minutes for LF-CAL and 15 minutes for LF-CHR).

\section{Characteristics of the patients}

In patients with UC, $62.8 \%$ were male and the mean age at the time of testing was $48.4 \pm 13.2$ years. The mean follow-up period was $17.2 \pm 6.3$ months. The mean partial Mayo score was $3.2 \pm 2.2$. The mean FC-ELISA and FC-QPOCT levels were 906.3 $\pm 1,484.9$ and $529.0 \pm 682.8$ $\mu \mathrm{g} / \mathrm{g}$, respectively.

In patients with $\mathrm{CD}, 63.2 \%$ were male and the mean age at the time of testing was $34.5 \pm 13.0$ years. The mean 
CDAI was $148.2 \pm 96.5$. The mean FC-ELISA and FCQPOCT levels were 1,054.1 \pm 1,252.5 and 579.0 \pm 646.0 $\mu \mathrm{g} / \mathrm{g}$, respectively. Regarding disease location, ileal CD was most common (53\%), followed by ileocolonic (39\%) and colonic $\mathrm{CD}(8 \%)$. Involvement of the upper gastrointestinal tract was present in only two patients. Patients' characteristics at the time of inclusion are summarized in Table 1.

\section{The correlation of FC with clinical activity and sero- logic markers}

The partial Mayo score and FC-ELISA levels showed a significant correlation in UC patients $(r=0.541, p=0.000)$ (Fig. 1A). In addition, there was significant correlation between the CDAI and FC-ELISA levels in CD patients $(r$ $=0.520, p=0.001$ ) (Fig. 1B).

In patients with UC, the FC-ELISA levels significantly correlated with the WBC $(r=0.314, p=0.020)$ and CRP $(r$ $=0.328, p=0.014)$, but not with the hematocrit $(r=0.174, p$ $=0.203)$. In contrast, FC-QPOCT levels only significantly correlated with CRP $(r=0.395, p=0.023)$. In patients with $\mathrm{CD}$, the FC-ELISA levels significantly correlated with $\mathrm{CRP}(r=0.358, p=0.027)$ and the hematocrit $(r=0.322, p$ $=0.049)$, but not with the WBC $(r=0.303, p=0.858)$. FCQPOCT also significantly correlated with CRP $(r=0.358$, $p=0.027)$ and hematocrit $(r=0.322, p=0.049)$.

In patients with UC, a cutoff value of $211.3 \mu \mathrm{g} / \mathrm{g}$ for the FC-ELISA had a sensitivity of $77.4 \%$ and a specificity of $75.0 \%$ for the prediction of clinical remission with an AUC value of 0.80 (Fig. 2A). A cutoff value of $180.5 \mu \mathrm{g} / \mathrm{g}$ for the FC-QPOCT had a sensitivity of $81.3 \%$ and a specificity of $73.7 \%$, with an AUC value of 0.79 (Fig. 2B). In patients with CD, a cutoff value of $710 \mu \mathrm{g} / \mathrm{g}$ for the FC-ELISA had a sensitivity of $62.5 \%$ and a specificity of $59.1 \%$

Table 1. Patients' characteristics

\begin{tabular}{|c|c|c|}
\hline Variable & Ulcerative colitis & Crohn's disease \\
\hline No. of patients & 55 & 38 \\
\hline Male sex & $34(62.8)$ & $24(63.2)$ \\
\hline Age, yr & $48.4 \pm 13.2(23-80)$ & $34.5 \pm 13.0(18-70)$ \\
\hline Disease duration, yr & $6.7 \pm 4.8$ & $6.6 \pm 6.3$ \\
\hline Follow-up period, mon & $17.2 \pm 6.3$ & $15 \cdot 4 \pm 7 \cdot 5$ \\
\hline Disease location, $\mathrm{L} 1 / \mathrm{L}_{2} / \mathrm{L}_{3}$ & - & $20 / 3 / 15(53 / 8 / 39)$ \\
\hline Disease extension, $\mathrm{E}_{1} / \mathrm{E}_{2} / \mathrm{E}_{3}$ & $17 / 29 / 9(31 / 53 / 16)$ & - \\
\hline Disease behavior, $\mathrm{B}_{1} / \mathrm{B}_{2} / \mathrm{B}_{3}$ & - & $22 / 8 / 8(58 / 21 / 21)$ \\
\hline \multicolumn{3}{|l|}{ Current medication } \\
\hline 5 -ASA & 53 & 30 \\
\hline Systemic corticosteroids & 11 & 17 \\
\hline Azathiopurine & 16 & 29 \\
\hline TNF- $\alpha$ inhibitor & 7 & 9 \\
\hline Partial Mayo score & $3.2 \pm 2.2$ & - \\
\hline Crohn's disease activity index & - & $148.2 \pm 96.5$ \\
\hline \multicolumn{3}{|l|}{ Serologic marker } \\
\hline WBC, g/L & $7,575.1 \pm 3,390.3$ & $5,292.9 \pm 1,640.2$ \\
\hline Hematocrit, \% & $40.8 \pm 3 \cdot 3$ & $39.0 \pm 4.0$ \\
\hline C-reactive protein, mg/dL & $0.7 \pm 2.4$ & $0.6 \pm 1.0$ \\
\hline FC-ELISA level, $\mu \mathrm{g} / \mathrm{g}$ & $906.3 \pm 1,484.9$ & $1,054.1 \pm 1,252.5$ \\
\hline FC-QPOCT level, $\mu \mathrm{g} / \mathrm{g}$ & $529.0 \pm 682.8$ & $579.0 \pm 646.0$ \\
\hline
\end{tabular}

Values are presented as number $(\%)$ or mean $\pm \mathrm{SD}$ (range), or mean $\pm \mathrm{SD}$.

5-ASA, 5-aminosalicylic acid; TNF, tumor necrosis factor; WBC, white blood cell; FC, fecal calprotection; ELISA, enzyme-linked immunosorbent assay; QPOCT, quantitative point-of-care test. 
for the prediction of clinical remission with an AUC of 0.69 (Fig. 2C). However, FC-QPOCT did not present a satisfactory cutoff value.

\section{The correlation of FC with endoscopic activity}

Of the 55 patients with UC, 29 underwent endoscopy. Six colonoscopies and 23 sigmoidoscopies were performed by expert endoscopists who have performed more than 1,000 colonoscopies. Of the 38 patients with CD, only three underwent colonoscopy. Thus, we could not obtain reliable data regarding the correlation between FC and endoscopic activity in CD patients.

FC-ELISA levels showed a significant correlation with the MES $(r=0.564, p=0.001)$. In contrast, there was no significant correlation between FC-QPOCT and endoscopic activity $(r=0.382, p=0.130)$.

According to the MES, seven patients (24\%) had endoscopic remission, seven (24\%) had mid activity, nine (31\%) had moderate activity, and six (21\%) had severe activity. The mean FC-ELISA level was significantly lower in the endoscopic remission group (MES of o) compared with the endoscopically active group $(85.5 \pm 55.6 \mu \mathrm{g} / \mathrm{g}$ vs.
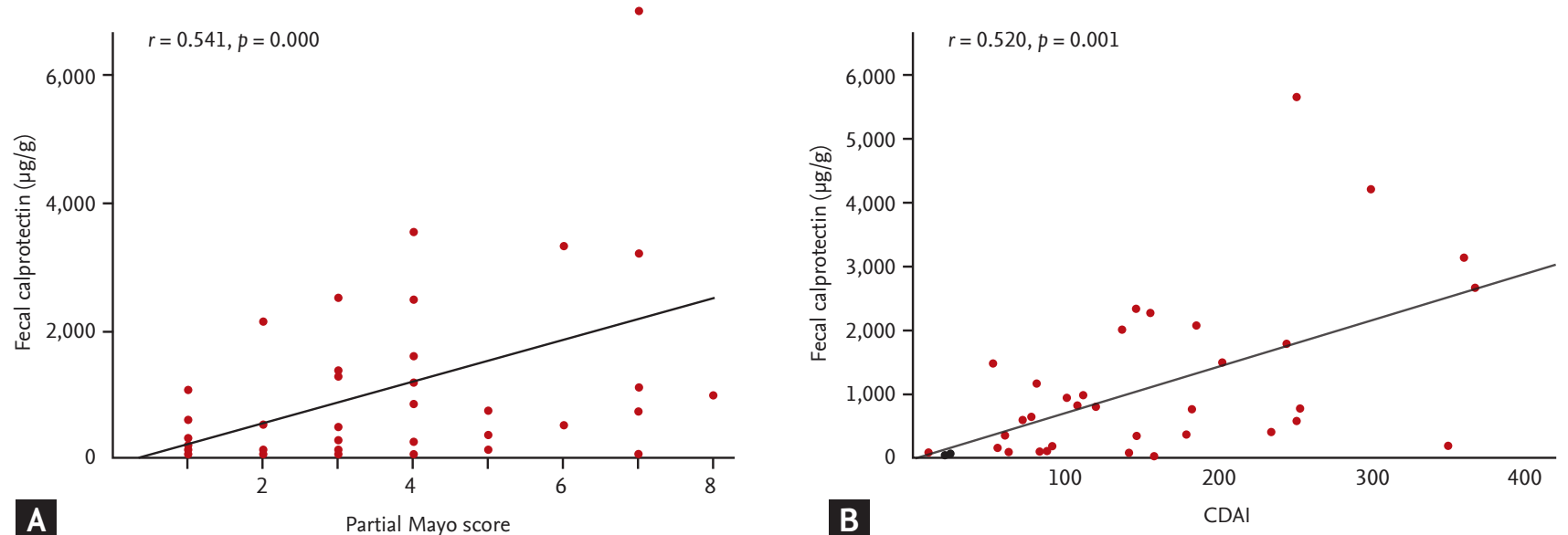

Figure 1. Correlations between clinical activity with FC-ELISA levels. Clinical indices and FC-ELISA shows significant correlation in both ulcerative colitis (A) and Crohn's disease (B). FC, fecal calprotection; ELISA, enzyme-linked immunosorbent assay; CDAI, Crohn's disease activity index.
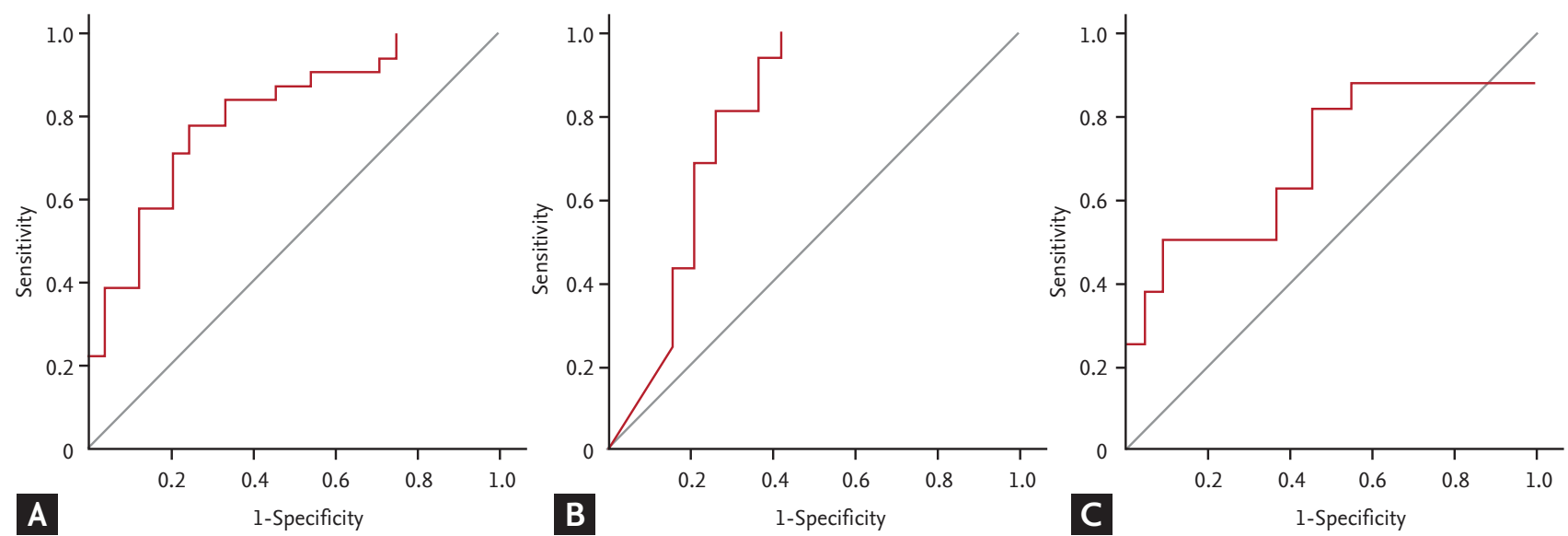

Figure 2. Receiver operating characteristic curves of fecal calprotection (FC) in relation to clinical remission. (A) FC-ELISA test in ulcerative colitis (UC) (sensitivity 77.4\%; specificity 75.0\% at FC-ELSIA 211.3 $\mu \mathrm{g} / \mathrm{g}$; AUC, 0.80). (B) FC-QPOCT in UC (sensitivity 81.3\%; specificity 73.7\% at FC-QPOCT 180.5 $\mu \mathrm{g} / \mathrm{g}$; AUC, 0.79). (C) FC-ELISA test in Crohn's disease (sensitivity 62.5\%; specificity 59.1\% at FC-ELSIA $710 \mu \mathrm{g} / \mathrm{g}$; AUC, o.69). ELISA, enzyme-linked immunosorbent assay; AUC, area under the curve; QPOCT, quantitative point-of-care test. 
$1,503.7 \pm 2,129.9 \mu \mathrm{g} / \mathrm{g}, p=0.005$ ) (Fig. 3).

In UC, a cutoff value of $201.0 \mu \mathrm{g} / \mathrm{g}$ for the FC-ELISA had a sensitivity of $81.8 \%$ and a specificity of $100 \%$ for the prediction of endoscopic remission with an AUC of 0.88. For FC-QPOCT, a cutoff value of $150.5 \mu \mathrm{g} / \mathrm{g}$ predicted endoscopic remission with a sensitivity of $85.7 \%$ and a specificity of $100 \%$. In the multivariate analysis, there were no independent variables significantly related to endoscopic remission.

\section{The correlation between FC-ELISA and FC-QPOCT}

FC levels measured by ELISA and QPOCT showed very

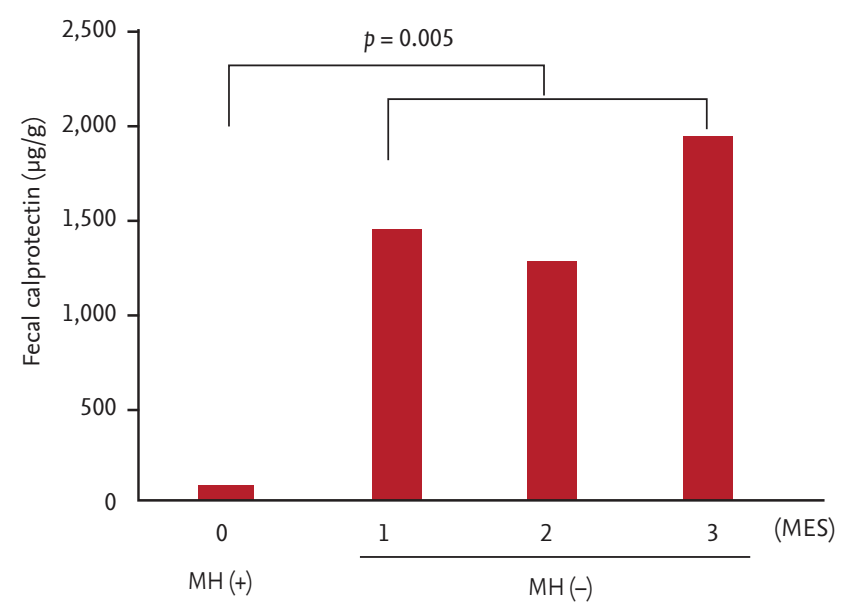

Figure 3. Fecal calprotectin-enzyme-linked immunosorbent assay (FC-ELISA) levels according to Mayo endoscopic subscore (MES). FC-ELISA levels were significantly lower in mucosal healing group compared with endoscopically active group. $\mathrm{MH}$, mucosal healing.

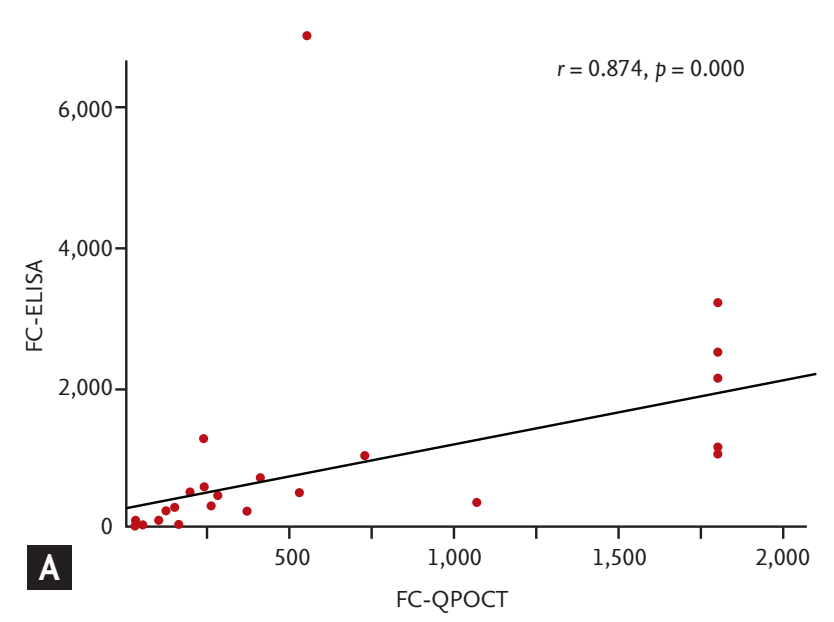

close correlation in both UC $(r=0.874, p=0.000)$ and CD $(r=0.908, p=0.000)$ (Fig. 4).

\section{DISCUSSION}

The current study evaluated the usefulness of FC as a biomarker for monitoring disease activity in patients with IBD using both ELISA and QPOCT. We confirmed that FC level was strongly associated with disease activity indices, serologic markers, and endoscopic activity in patients with IBD. In particular, both calprotectin assays could predict $\mathrm{MH}$ with high sensitivity (> 81\%) and specificity (100\%) in UC patients. Therefore, FC may be a useful alternative to repeated endoscopies. In addition, QPOCT can be used more conveniently than ELISA to assess FC in clinical practice.

Previous studies have demonstrated that $\mathrm{MH}$ is intimately related to a favorable clinical course of IBD in terms of treatment response, relapse, hospitalization, and surgery $[4,22,23]$. Colonoscopy is still considered the gold standard for the evaluation of mucosal inflammation, but is invasive, time-consuming and uncomfortable for patients. Therefore, an interest in noninvasive and reliable biomarkers of mucosal inflammation that have comparable performance to colonoscopy exists in clinical practice.

Several studies have previously suggested FC as a surrogate marker for endoscopic disease activity in IBD patients. However, some studies have shown contrary

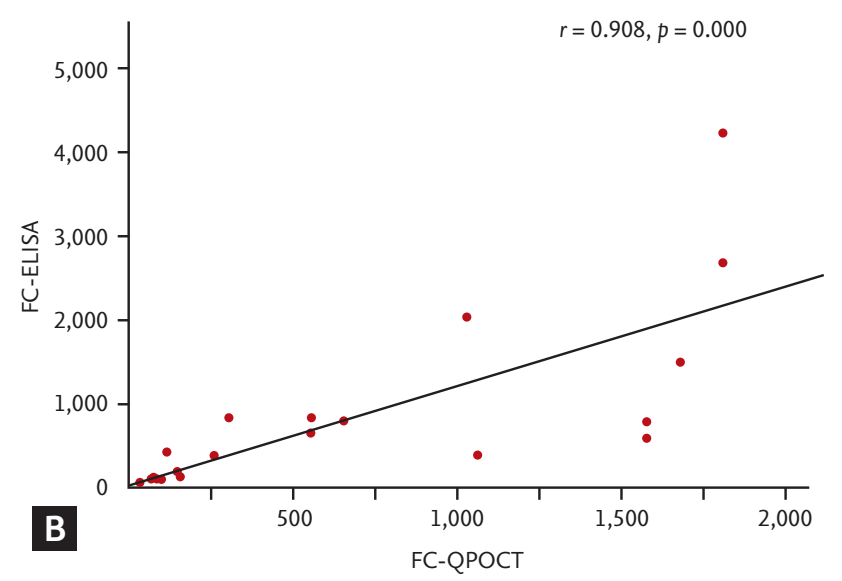

Figure 4. Correlations between FC-ELISA and FC-QPOCT levels in ulcerative colitis (UC) and Crohn's disease (CD). FC-ELISA and FC-QPOCT showed very close correlation in both UC (A) and CD (B). FC, fecal calprotection; ELISA, enzyme-linked immunosorbent assay; QPOCT, quantitative point-of-care test. 
results [7,11,24-26]. A Belgian study showed a significant correlation between FC levels and endoscopic activity in both UC and CD [7]. In a small population-based cohort study, FC was significantly related to the endoscopic activity score in patients with IBD [27]. A Spanish study showed that FC predicts endoscopic remission (Crohn's Disease Endoscopic Index of Severity [CDEIS] < 3) and postoperative endoscopic recurrence in CD patients [15]. In contrast, Denis et al. [26] failed to find a significant correlation between CDEIS and FC in symptomatic CD patients with normal CRP levels. In addition, there are still numerous controversial issues. The current data are not yet conclusive enough to establish an FC cutoff level as a predictor of $\mathrm{MH}$ or postoperative recurrence. The published cut-off values for predicting mucosal inflammation varies from 50 to $250 \mu \mathrm{g} / \mathrm{g}$. Xiang et al. [25] showed that FC with a $50 \mu \mathrm{g} / \mathrm{g}$ cutoff level had a specificity of $79.4 \%$ and a sensitivity of $91.9 \%$ in the discrimination of active and inactive disease according to the Sutherland score. Schoepfer et al. [11] illustrated that with a $100 \mathrm{mg} / \mathrm{L}$ cutoff level, FC predicted endoscopic activity as defined by a Rachmilewitz index $\geq 4$ with a specificity of $88 \%$ and a sensitivity of $86 \%$. Recently, D'Haens et al. [7] found a $250 \mu \mathrm{g} / \mathrm{g}$ cutoff level to be the most accurate for the prediction of the presence of any mucosal inflammation in patients with UC as defined by a Mayo endoscopic sub-score $\geq 1$ with a specificity of $100 \%$ and a sensitivity of $71 \%$ as well as for the prediction of the presence of large ulcers in CD. In line with these results, we demonstrated that FC with a $201.3 \mu \mathrm{g} / \mathrm{g}$ cutoff level for the FC-ELISA or a $150.5 \mathrm{\mu g} / \mathrm{g}$ cutoff level for the FC-QPOCT is the most accurate for the prediction of any endoscopic activity as defined by a MES > 0 .

Although the FC-ELISA method is the most commonly used method to measure FC, recent studies have been conducted to evaluate the accuracy of the FC-QPOCT in predicting disease activity in IBD. Lobaton et al. [15] demonstrated that, in patients with $\mathrm{CD}$ with ileocolonic resection, the prediction of endoscopic remission using FC-QPOCT presented an AUC of 0.933. The same authors showed that the prediction of endoscopic remission with FC-QPOCT presented an AUC of 0.906 in patients with UC [12]. The current study also explored the validity of the FC-QPOCT compared with the FC-ELISA method. Our results suggest that the FC-QPOCT is comparable to the FC-ELISA in the prediction of disease activity in IBD. The FC-QPOCT is easy to perform and able to produce more rapid quantitative results than the FC-ELISA method. Therefore, the FC-QPOCT appears to be more suitable for real practice and could be more widely used in the future.

Our study has several limitations. First, there was a lack of follow-up data for FC in our patients. In order to show the close correlation between FC and disease activity, FC data taken at different time points with varying degree of disease activity in the same patient may be more useful and informative. Second, the number of patients included in our study was relatively small. Third, we did not assess histological findings. The definition of $\mathrm{MH}$ is still controversial and some researchers suggest that $\mathrm{MH}$ should include not only endoscopic healing but also the histological absence of mucosal inflammation $[28,29]$. Recently, Theede et al. [30] illustrated that the FC level correlated with endoscopic and histologic inflammatory activity in 120 UC patients who had endoscopic and histologic features of mucosal healing (positive predictive value 0.71 and 0.75 , negative predictive value 0.65 and 0.90 ). Thus, future studies should evaluate the relationship between FC and histologic inflammation. Fourth, since colonoscopy was performed in too few CD patients, the correlation between FC and endoscopic activity in CD could not be assessed. Lastly, as showed in Fig. 1B, higher fraction of our CD patients showed relatively low disease activity $(\mathrm{CDAI}<220)$. This uneven distribution could lead selection biases.

In conclusion, our results indicated that FC is a reliable surrogate marker of endoscopic activity in IBD. FC could be especially useful in the prediction of endoscopic remission in patients with UC. Therefore, FC has the potential to replace colonoscopy for the serial assessment of mucosal inflammation in IBD patients. In addition, the excellent correlation observed between the FC-ELISA and the rapid QPOCT allows a more easy assessment of FC in clinical practice.

\section{KEY MESSAGE}

1. Fecal calprotectin (FC) level was strongly associated with disease activity indices, serologic markers, and endoscopic activity in patients with inflammatory bowel disease. 
2. In particular, calprotectin assays could predict mucosal healing with high sensitivity $(>81 \%)$ and specificity $(100 \%)$ in ulcerative colitis patients. Therefore, FC may be a useful alternative to repeated endoscopies.

3. Quantitative point-of care test can be used more conveniently than enzyme-linked immunosorbent assay to assess FC in clinical practice.

\section{Conflict of interest}

No potential conflict of interest relevant to this article was reported.

\section{REFERENCES}

1. Neurath MF, Travis SP. Mucosal healing in inflammatory bowel diseases: a systematic review. Gut 2012;61:1619-1635.

2. Sakuraba A, Annunziata ML, Cohen RD, Hanauer SB, Rubin DT. Mucosal healing is associated with improved long-term outcome of maintenance therapy with natalizumab in Crohn's disease. Inflamm Bowel Dis 2013;19:2577-2583.

3. Asgharpour A, Cheng J, Bickston SJ. Adalimumab treatment in Crohn's disease: an overview of long-term efficacy and safety in light of the EXTEND trial. Clin Exp Gastroenterol 2013;6:153-160.

4. Froslie KF, Jahnsen J, Moum BA, Vatn MH; IBSEN Group. Mucosal healing in inflammatory bowel disease: results from a Norwegian population-based cohort. Gastroenterology 2007;133:412-422.

5. Sipponen T, Kolho KL. Fecal calprotectin in diagnosis and clinical assessment of inflammatory bowel disease. Scand J Gastroenterol 2015;50:74-80.

6. Smith LA, Gaya DR. Utility of faecal calprotectin analysis in adult inflammatory bowel disease. World J Gastroenterol 2012;18:6782-6789.

7. D'Haens G, Ferrante M, Vermeire S, et al. Fecal calprotectin is a surrogate marker for endoscopic lesions in inflammatory bowel disease. Inflamm Bowel Dis 2012;18:2218-2224.

8. Sipponen T, Savilahti E, Karkkainen P, et al. Fecal calprotectin, lactoferrin, and endoscopic disease activity in monitoring anti-TNF-alpha therapy for Crohn's disease.
Inflamm Bowel Dis 2008;14:1392-1398.

9. Sipponen T, Bjorkesten CG, Farkkila M, Nuutinen H, Savilahti E, Kolho KL. Faecal calprotectin and lactoferrin are reliable surrogate markers of endoscopic response during Crohn's disease treatment. Scand J Gastroenterol 2010;45:325-331.

10. D'Inca R, Dal Pont E, Di Leo V, et al. Calprotectin and lactoferrin in the assessment of intestinal inflammation and organic disease. Int J Colorectal Dis 2007;22:429-437.

11. Schoepfer AM, Beglinger C, Straumann A, Trummler M, Renzulli P, Seibold F. Ulcerative colitis: correlation of the Rachmilewitz endoscopic activity index with fecal calprotectin, clinical activity, C-reactive protein, and blood leukocytes. Inflamm Bowel Dis 2009;15:1851-1858.

12. Lobaton T, Rodriguez-Moranta F, Lopez A, Sanchez E, Rodriguez-Alonso L, Guardiola J. A new rapid quantitative test for fecal calprotectin predicts endoscopic activity in ulcerative colitis. Inflamm Bowel Dis 2013;19:1034-1042.

13. Schoepfer AM, Beglinger C, Straumann A, et al. Fecal calprotectin more accurately reflects endoscopic activity of ulcerative colitis than the Lichtiger Index, C-reactive protein, platelets, hemoglobin, and blood leukocytes. Inflamm Bowel Dis 2013;19:332-341.

14. Lasson A, Simren M, Stotzer PO, Isaksson S, Ohman L, Strid H. Fecal calprotectin levels predict the clinical course in patients with new onset of ulcerative colitis. Inflamm Bowel Dis 2013;19:576-581.

15. Lobaton T, Lopez-Garcia A, Rodriguez-Moranta F, Ruiz A, Rodriguez L, Guardiola J. A new rapid test for fecal calprotectin predicts endoscopic remission and postoperative recurrence in Crohn's disease. J Crohns Colitis 2013;7:e641-e651.

16. Wassell J, Wallage M, Brewer E. Evaluation of the Quantum Blue rapid test for faecal calprotectin. Ann Clin Biochem 2012;49(Pt 1):55-58.

17. Silverberg MS, Satsangi J, Ahmad T, et al. Toward an integrated clinical, molecular and serological classification of inflammatory bowel disease: report of a Working Party of the 2005 Montreal World Congress of Gastroenterology. Can J Gastroenterol 2005;19 Suppl A:5A-36A.

18. Schroeder KW, Tremaine WJ, Ilstrup DM. Coated oral 5-aminosalicylic acid therapy for mildly to moderately active ulcerative colitis: a randomized study. N Engl J Med 1987;317:1625-1629.

19. Best WR, Becktel JM, Singleton JW, Kern F Jr. Development of a Crohn's disease activity index: National 
Cooperative Crohn's Disease Study. Gastroenterology 1976;70:439-444.

20. Daperno M, D'Haens G, Van Assche G, et al. Development and validation of a new, simplified endoscopic activity score for Crohn's disease: the SES-CD. Gastrointest Endosc 2004;60:505-512.

21. Schoepfer AM, Beglinger C, Straumann A, et al. Fecal calprotectin correlates more closely with the Simple Endoscopic Score for Crohn's disease (SES-CD) than CRP, blood leukocytes, and the CDAI. Am J Gastroenterol 2010;105:162-169.

22. Peyrin-Biroulet L, Ferrante M, Magro F, et al. Results from the 2nd Scientific Workshop of the ECCO. I. Impact of mucosal healing on the course of inflammatory bowel disease. J Crohns Colitis 2011;5:477-483.

23. Colombel JF, Rutgeerts P, Reinisch W, et al. Early mucosal healing with infliximab is associated with improved long-term clinical outcomes in ulcerative colitis. Gastroenterology 2011;141:1194-1201.

24. Roseth AG, Aadland E, Grzyb K. Normalization of faecal calprotectin: a predictor of mucosal healing in patients with inflammatory bowel disease. Scand J Gastroenterol 2004;39:1017-1020.

25. Xiang JY, Ouyang Q, Li GD, Xiao NP. Clinical value of fecal calprotectin in determining disease activity of ulcerative colitis. World J Gastroenterol 2008;14:53-57.

26. Denis MA, Reenaers C, Fontaine F, Belaiche J, Louis E. Assessment of endoscopic activity index and biological inflammatory markers in clinically active Crohn's disease with normal C-reactive protein serum level. Inflamm Bowel Dis 2007;13:1100-1105.

27. Ricanek P, Brackmann S, Perminow G, et al. Evaluation of disease activity in IBD at the time of diagnosis by the use of clinical, biochemical, and fecal markers. Scand J Gastroenterol 2011;46:1081-1091.

28. Bessissow T, Lemmens B, Ferrante M, et al. Prognostic value of serologic and histologic markers on clinical relapse in ulcerative colitis patients with mucosal healing. Am J Gastroenterol 2012;107:1684-1692.

29. Rosenberg L, Nanda KS, Zenlea T, et al. Histologic markers of inflammation in patients with ulcerative colitis in clinical remission. Clin Gastroenterol Hepatol 2013;11:991-996.

30. Theede K, Holck S, Ibsen P, Ladelund S, Nordgaard-Lassen I, Nielsen AM. Level of fecal calprotectin correlates with endoscopic and histologic inflammation and identifies patients with mucosal healing in ulcerative colitis. Clin Gastroenterol Hepatol 2015;13:1929-1936. 